www.jmscr.igmpublication.org

Impact Factor 5.84

Index Copernicus Value: 71.58

ISSN (e)-2347-176x ISSN (p) 2455-0450

crossref DOI:_https://dx.doi.org/10.18535/jmscr/v6i1.01

Journal Of Medical Science And Clinical Research

\title{
The Added Diagnostic Value of Transvaginal Ultrasound to Transabdominal Ultrasound in Detection and Assessment of Placenta Previa
}

\author{
Authors \\ Ola Mohamed Darwish ${ }^{1}$, Alaa Eldin Mohamed Mustafa ${ }^{2}$ \\ ${ }^{1}$ Department of Radio-Diagnosis, Hattah Hospital, Dubai Health Authority, Dubai, UAE \\ ${ }^{2}$ Department of Radio-diagnosis, Faculty of Medicine, Alexandria University, Egypt
}

\begin{abstract}
Aim: To evaluate the added diagnostic value of the transvaginal ultrasound (TVUS) to transabdominal ultrasound $(T A U S)$ in the detection and evaluation of placenta praevia (PP).

Methods: This study was carried out on 46 pregnant women during the second trimester, the third trimester or both, over two year's duration. The study group was divided into two main groups: a- The pathological group including 8 pregnant women who were diagnosed to have abnormal relation of their placentae to the internal os in the $2^{\text {nd }}$ trimester. Only two of them were diagnosed to have PP in the third trimester and the remaining six were excluded from the study $b$-The control group which included 20 pregnant women who were diagnosed to have normal relation of their placentae to the internal os by both TAUS and TVUS. The findings of both techniques were compared with each other and with the operative results after cesarean sections (CS). Statistical analysis was carried out using Chi-square test, Fisher's Exact or Monte Carlo correction \& Student t-test.
\end{abstract}

Results: TAUS correctly diagnosed 12/12 (100\%) cases of PP totalis, 2/2 cases of PP near totalis, 3/4 cases of PP marginalis. While it overestimated one case of PP marginalis as PP totalis, in addition to false positive diagnosis of $P P$ in two cases who were falsely diagnosed as PP marginalis in one case and low lying placenta in another case. Both cases were diagnosed as normal by TVUS and operative room data. TAUS succeeded in negation of PP in all 20 (100\%) control cases. It showed 100\%, 90\%, 90\% and 100\% sensitivity, specificity, positive predictive value and negative predictive values respectively. TVUS showed true positive diagnosis of PP in 100\% of cases, and negated PP in all 20 control pregnant women (true negative diagnosis). It correctly excluded the false positive diagnosis in two cases as low lying placenta and PP marginalis suggested by TAUS. It also corrected another overestimated diagnosis of PP totalis in a case of PP marginalis by TAUS. It showed 100\% diagnostic accuracy, sensitivity, specificity, positive predictive value \& negative predictive value.

Conclusion: Diagnosis of PP should not be established except on the $3^{\text {rd }}$ trimester. TAUS has higher incidence of false positive diagnosis of PP in comparison to TVUS. TVUS is a valuable procedure to compliment TAUS in the evaluation of patients with suspected PP.

Keywords: Transabdominal ultrasound, Transvaginal ultrasound, Placenta previa.

\section{Introduction}

Placenta previa is an obstetric complication in which the placenta is inserted partially or totally in lower uterine segment. ${ }^{1}$ It is one of the most serious causes of vaginal bleeding during pregnancy. ${ }^{2}$ Four grades of placenta previa are known: low lying, marginalis, near totalis and totalis. ${ }^{2,5}$ 
The exact etiology of placenta previa is unknown. ${ }^{3}$ Ultrasound is the first modality in diagnosis \& assessment of placenta previa using transabdominal \& transvaginal techniques. Although MRI has been found to be superior to sonography but its high cost \& limited availability make the technique not popular. ${ }^{4}$

Placenta previa is an obstetric complication in which the placenta is inserted partially or totally in the lower uterine segment. ${ }^{1}$ It is a leading cause of vaginal bleeding during pregnancy. ${ }^{2}$ Four grades of placenta previa are known: low lying, marginalis, near totalis and totalis. ${ }^{2,5}$ Exact etiology of placenta praevia is unknown. It is hypothesized to be related to abnormal endometrial vascularity caused by scarring or atrophy from previous trauma, surgery, or infection. ${ }^{3}$ Previa can be confirmed with transabdominal ultrasound and transvaginal ultrasound. ${ }^{4}$ Transvaginal ultrasound has the advantage of being accurate. It is also proven to be safe. However, many obstacles to its use are defined like fear of the patients to develop bleeding or abortion and as well as the traditions and habits in some cultures. Transabdominal ultrasound has advantages of being easy, very safe and accepted traditionally. It also has some limitations like: obesity and false positive results. ${ }^{4}$

\section{Aim}

To evaluate the added diagnostic value of the transvaginal ultrasound (TVUS) to transabdominal ultrasound (TAUS) in the detection and evaluation of placenta praevia (PP).

\section{Materials and Methods}

This study was carried out on 46 pregnant women during the second trimester, the third trimester or both, referred to Alshat by university hospital, Faculty of Medicine, Alexandria university, over a near two years duration. The 46 pregnant women were divided into two main groups: a- The pathological group which included 8 pregnant women who were diagnosed to have abnormal relation of their placentae to the internal os in the $2^{\text {nd }}$ trimester \& underwent follow up in the $3^{\text {rd }}$ trimester (only two of them were diagnosed to have placenta previa in the follow up third trimester US evaluation. Subsequently, the remaining six cases were excluded from the study.. The pathological group included other 18 pregnant women who were diagnosed to have placenta previa by TAUS, TVUS or both in the third trimester b- The control group which included 20 pregnant women who were diagnosed to have normal placenta as regard the relation to the internal os by TAUS \& TVUS.

Examination was done by real time ultrasonography. Ultrasound machines were mainly Toshiba XARIO 200 \& GE LOGIQ B6. The transabdominal probes frequencies were $6 \mathrm{Mhz} \& 5 \mathrm{Mhz}$ for both machines respectively. The transvaginal probes frequencies were $8 \mathrm{Mhz}$ $\& 10 \mathrm{Mhz}$ for both machines respectively.

Transvaginal probe was gently introduced for about $3-4 \mathrm{~cm}$ beyond the introitus. Distance between internal os and lower edge of placenta was measured. The diagnosis of placenta previa was made if placental edge was located within $2 \mathrm{~cm}$ of internal os. TAUS was performed with convex linear transducer. Bladder was kept semifull during the examination. The transducer was positioned directly on the supra pubic region in sagittal orientation, delineating the relation of the placental edge to the internal os.

The classification system propsed by Farine $1988^{(5)}$ regarding grading of placenta previa was followed including:

Normal: distance between lower placental edge and internal os is $>2 \mathrm{~cm}$, Low lying: the distance ranges from $0.5-2 \mathrm{~cm}$, Marginalis: lower placental edge is just touching the internal os border, Near totalis: the placental edge is incompletely covering the internal os, and Totalis: where the lower placental edge is totally covering the internal os.

Other US findings were collected and tabulated including placental position, grade, separation, presence of absence of retro-placental hematoma and placental inversion 
TVUS was only capable of examination of the lower uterine segment and detection of the relation of the lower edge of the placenta to the internal os applying the same parameters.

The presence or absence of placenta previa was confirmed at delivery. Absence of clinically significant placenta previa at delivery was confirmed, if no placental tissue was seen overlying the cervix at caesarean section or when the distance from lower edge of placenta to internal cervical os was more than $2 \mathrm{~cm}$.

Statistical analysis was carried out using IBM SPSS software package version 20.0 Comparison between different groups regarding catigorial variables was tested using Chi-square test, Fisher's Exact or Monte Carlo correction \& Student t-test.

\section{Results}

The study included 20 pregnant women belonged to placenta previa group \& other 20 pregnant women belonged to the control group. The ages of the studied pregnant women, belonged to the two groups, ranged from 25 to 40 years old. The mean age was $34.50 \pm 3.27$ for the placenta previa group $\&$ was $30 \pm 3.54$ for the control group. Increasing maternal age was found to be statistically significant as a risk factor of placenta previa $(p=0.024)$

Table 1: Comparison between placenta previa group $(n=20) \&$ control group $(n=20)$ regarding age distribution:

\begin{tabular}{|c|c|c|c|c|c|}
\hline $\begin{array}{l}\text { Age } \\
\text { groups }\end{array}$ & \multicolumn{2}{|c|}{$\begin{array}{c}\text { Placenta } \\
\text { previa } \\
\text { group }\end{array}$} & \multicolumn{2}{|c|}{$\begin{array}{c}\text { Control } \\
\text { group }\end{array}$} & ${ }^{M C_{p}}$ \\
\hline & $\begin{array}{c}\text { Num } \\
\text { ber }\end{array}$ & $\begin{array}{l}\text { Perce } \\
\text { ntage }\end{array}$ & $\begin{array}{c}\text { Num } \\
\text { ber }\end{array}$ & $\begin{array}{l}\text { Perce } \\
\text { ntage }\end{array}$ & \\
\hline $25<30$ & 2 & $10 \%$ & 8 & $40 \%$ & $0.024 *$ \\
\hline $30<35$ & 8 & $40 \%$ & 9 & $45 \%$ & \\
\hline $35<40$ & 10 & $50 \%$ & 3 & $15 \%$ & \\
\hline Total & 20 & $100 \%$ & 20 & $100 \%$ & \\
\hline
\end{tabular}

Painless vaginal bleeding was the main complaint in 11 out of 20 pregnant women $(55 \%$ of the placenta previa group). Two out of 11 them presented with profuse vaginal bleeding. Vaginal spotting was the main complaint in $9 / 11$ pregnant women. Contrarily, there were only 2/20 (10\%) pregnant women presented by vaginal bleeding (vaginal spotting) among the control group. The remaining 18/20 pregnant women were asymptomatic (90\% of the control group). Vaginal bleeding was found to be statistically significant among placenta previa group, $\mathrm{P}$ value for Chi square test for comparing between the two groups $=0.002$

Table 2 shows a comparison between both groups as regards the number of previous gestations. The results were statistically significant regarding the incidence of placenta previa with increased parity. $\mathrm{P}$ value for Chi square test for comparing between the two groups $=0.040$ (Table 2)

Table 2: Comparison between placenta previa group ( $\mathrm{n}=20$ pregnant women) \& control group ( $\mathrm{n}=20$ pregnant women) as regards the number of gestations

\begin{tabular}{|c|c|c|c|c|c|c|}
\hline No $G$ & \multicolumn{2}{|c|}{$\begin{array}{l}\text { Previa } \\
\text { group }\end{array}$} & \multicolumn{2}{|c|}{$\begin{array}{l}\text { Control } \\
\text { group }\end{array}$} & $X^{2}$ & ${ }^{\mathrm{MC}} \mathrm{P}$ \\
\hline & No & $\%$ & No & $\%$ & \multirow{6}{*}{$9.88 *$} & \multirow{6}{*}{$0.040 *$} \\
\hline G1 & 1 & $5 \%$ & 4 & $20 \%$ & & \\
\hline G2 & 3 & $15 \%$ & 6 & $30 \%$ & & \\
\hline G3 & 4 & $20 \%$ & 7 & $35 \%$ & & \\
\hline G4 & 5 & $25 \%$ & 1 & $5 \%$ & & \\
\hline G5 & 7 & $35 \%$ & 2 & $10 \%$ & & \\
\hline
\end{tabular}

$\chi^{2}, \chi^{2}$ and $\mathrm{p}$ values for Chi square test for comparing between the two groups.

${ }_{\mathrm{MC}} \mathrm{p}$ : $\mathrm{p}$ value for Monte Carlo for Chi square test for comparing between the two groups.

$*$ : Statistically significant at $\mathrm{p} \leq 0.05$.

As regards fetal biophysical profile including fetal breath rate tone, muscle tone, and heart rate, were reported normal in all pregnant women belonged to both placenta previa and control groups. Only one pregnant female belonged to the placenta previa group expressed moderate oligohydramnios. Placenta previa showed no appreciable diverse effect on fetal well being.

All fetuses belonged to placenta previa group showed normal umbilical arteries \& middle cerebral arteries resistive indices, denoting absence diverse effect of placenta previa on placental and fetal circulations)

History of previous cesarean section was positive in $12 / 20(60 \%)$ pregnant women belonged to 
previa group compared to $4 / 20$ (20\%) women belonged to the control group. Findings were found to be statistically significant regarding the increased incidence of placenta previa with history or previous $\mathrm{CS}(\mathrm{p}=0.010)$. On the other hand the results were of insignificant values among other types of surgical interventions including previous $\mathrm{D} \& \mathrm{C}$, previous removal of endometrial polyps or previous myomectomies which were expressed by $40 \%, 10 \%$ and $5 \%$ of pregnant women belonged to the placenta previa group compared to $25 \%, 0 \%$ and $0 \%$ of pregnant women belonged to the control group respectively Final operative room diagnosis showed 12/10 cases of PP totalis, 2/20 cases of PP near totalis, $4 / 20$ cases with PP marginalis. No cases were reported as low lying placentae. Finally two cases were reported as normal relation of the lower pole of the placenta to the internal os.

TAUS correctly diagnosed 12/12 (100\%) cases of PP totalis, (figure 1\&2), 2/2 cases of PP near totalis, 3/4 cases of PP marginalis. It overestimated one case of PP marginalis and reported it as PP totalis. In addition to that it gave a false positive diagnosis of PP in two cases who were falsely diagnosed as PP marginalis in one case and low lying placenta in another case (figure 3). Both cases were diagnosed as normal by TVUS and operative room data. TAUS succeeded in negation of PP in all $20(100 \%)$ control cases. It showed $100 \%, 90 \%, 90 \%$ and $100 \%$ sensitivity, specificity, positive predictive value and negative predictive values respectively.

TVUS showed true positive diagnosis of PP in $100 \%$ of cases, and negated PP in all 20 control pregnant women (true negative diagnosis). It correctly excluded the false positive diagnosis in two cases as low lying placenta and PP marginalis suggested by TAUS. It also corrected another overestimated diagnosis of PP totalis in a case of PP marginalis by TAUS. It showed diagnostic accuracy, sensitivity, specificity, positive predictive value $\&$ negative predictive value of 100\%. (Tables $3 \& 4$ )
Table 3: diagnostic yields of TAUS Vs TVUS regarding the type of PP according to Farine description

\begin{tabular}{|c|c|c|c|c|c|}
\hline $\begin{array}{l}\text { Types of } \\
\text { placenta }\end{array}$ & \multicolumn{2}{|c|}{$\begin{array}{c}\text { Trans } \\
\text { abdominal US }\end{array}$} & \multicolumn{2}{|c|}{$\begin{array}{c}\text { Trans vaginal } \\
\text { US }\end{array}$} & $\begin{array}{l}\text { Operating } \\
\text { room results }\end{array}$ \\
\hline & $\begin{array}{l}\text { True } \\
\text { +ve }\end{array}$ & $\begin{array}{l}\begin{array}{l}\text { False } \\
+v e\end{array}\end{array}$ & $\begin{array}{l}\text { True } \\
+ \text { +ve }\end{array}$ & $\begin{array}{l}\begin{array}{l}\text { False } \\
+v e\end{array}\end{array}$ & \\
\hline PP Totalis & 12 & 1 & 12 & 0 & 12 \\
\hline $\begin{array}{l}\text { PP near } \\
\text { totalis }\end{array}$ & 2 & 0 & 2 & 0 & 2 \\
\hline $\begin{array}{l}\mathrm{PP} \\
\text { marginalis }\end{array}$ & 3 & 1 & 4 & 0 & 4 \\
\hline $\begin{array}{l}\text { Low lying } \\
\text { placenta }\end{array}$ & 0 & 1 & 0 & 0 & 0 \\
\hline Normal & 0 & 0 & 2 & 0 & 2 \\
\hline
\end{tabular}

Table 4: Statistical analysis of TAUS and TVUS diagnosis as confirmed by the operating room findings

\begin{tabular}{|l|c|c|c|c|c|}
\hline & $\begin{array}{c}\text { True } \\
+v e\end{array}$ & $\begin{array}{c}\text { True - } \\
\text { ve }\end{array}$ & $\begin{array}{c}\text { False } \\
+v e\end{array}$ & $\begin{array}{c}\text { False - } \\
\text { ve }\end{array}$ & Total \\
\hline TAUS & 18 & 20 & 2 & 0 & 40 \\
\hline TVUS & 18 & 22 & 0 & 0 & 40 \\
\hline
\end{tabular}

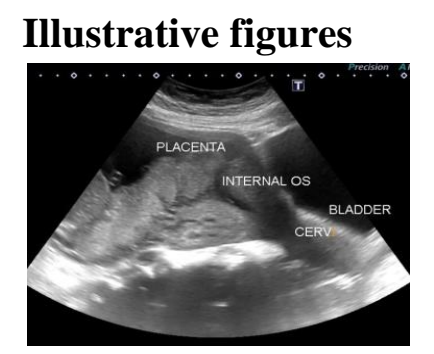

(a)

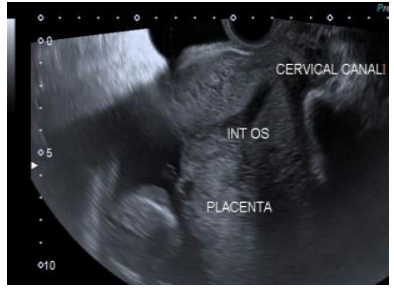

(b)
Figure (1): TAUS (a) \& TVUS (b): The placenta is totally covering the internal os by both techniques ......placenta previa totalis.

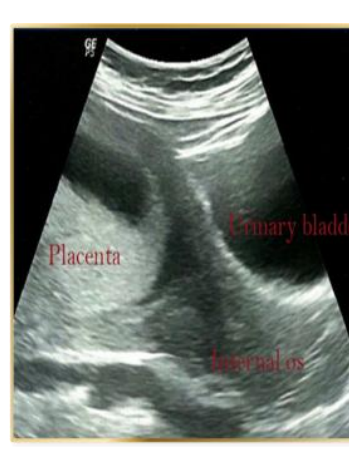

(a)

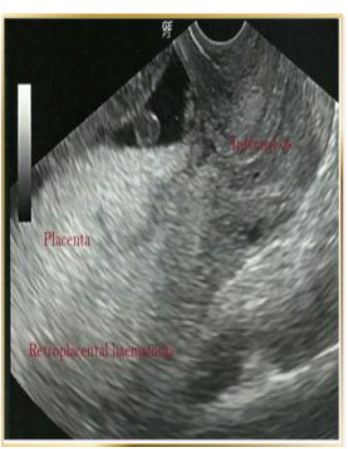

(b)
Figure 2: A G5P3A1, 32 years old pregnant woman who was presenting in the $3^{\text {rd }}$ trimester by profuse vaginal bleeding with history of previous 2 cesarean sections followed by D \& C for missed abortion 2 years prior to the examination. TAUS 
(a) and TVUS (b) showing placenta previa totalis with echogenic retro-placental hematoma. Findings were confirmed by operating room report.

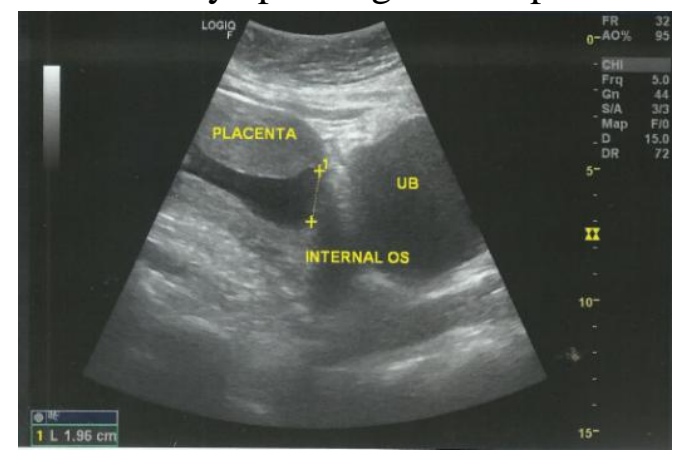

(a)

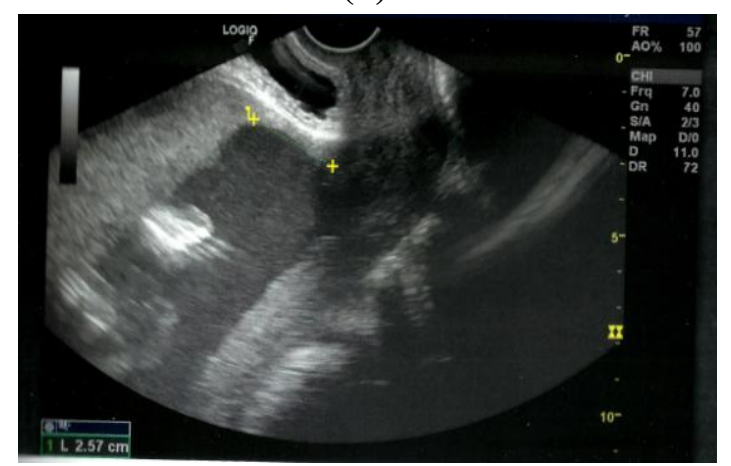

(b)

Figure (2): TAUS: The placenta is reported to be low lying, with its lower edge $19 \mathrm{~mm}$ from the internal os.(a) TVUS of the same pregnant woman shows that the placenta is completely normal in position with its lower edge is more than $2 \mathrm{~cm}$ from the internal os (b). False positive finding by TAUS

\section{Discussion}

The present study was carried out on 46 pregnant women over a period of two years. It aimed at assessing the added value of performing TVUS in detection and assessment of placenta previa. Only two out of 8 of pregnant females included in the current study who were examined in the $2^{\text {nd }}$ trimester and showed findings suggestive of PP, persisted to have placenta previa in the third trimester. Howards et al $(2014)^{6}$ reported only $1.6 \%$ incidence of persisted sonographic placenta previa or low lying placenta at or near term. She concluded that a low lying placenta sonographically diagnosed in the $2^{\text {nd }}$ trimester typically resolve by the $3^{\text {rd }}$ trimester and only rarely dose it persists to term or near term.
Similar findings have been reported by Blouin et al $(2012)^{7}$

The exact etiology of PP is unknown. The condition may me multi-factorial ${ }^{8-12,}$. It is postulated to be related to multiple risk factors including advancing maternal age (>35y), infertility treatment, muti-parity (5\% in rad multiparous women), previous uterine surgery, uterine insult or injury, previous cesarean delivery (including first pregnancy following previous cesarean delivery, previous or recurrent abortions, or previous placenta previa

In the current study, variable grades of PP were detected in 10 out of 20 PP group aged > 35 YO, with median age of $34 \mathrm{YO}$, this proved to be statistically significant $\quad(\mathrm{P}=0.024)$. Similarly, previous reports stated that increased maternal age, especially above 34 years old is associated with increased incidence of development of PP 11,13

In 2015, Rose said that Advancing maternal age appears to increase the risk of placenta previa independent of other risk factors. They reported that the incidence of placenta previa in the age group $\geq 35$ years $(3.6 \%)$ when compared to other age groups was found to be of statistically significant $(p<0.05){ }^{9}$

Currently, the incidence of associated of history of previous cesarean section and development of PP was detected in $60 \%$ of PP group, compared to only $20 \%$ of women belonged to the control group. This association was found to be statistically significant values among the women forming the current study group $(\mathrm{P}=0.001)$. Positive, linear correlation between PP and CS has been reported in the study of Ananth ${ }^{12}$. Similar incidences have been reported in the studies of Molosevic ${ }^{13}$, Itedal ${ }^{14}$ and David ${ }^{15}$. On the other hand, no greater disk of maternal complications in patient with four or more prior cesareans, including the risk of development of placenta previa except occurrence of abdominal adhesions have concluded in the study of Uyanikoglu $(2017)^{11}$ 
In the current study a significant relation between the incidence of PP and multiple previous gestations $(\mathrm{P}=0.040)$. Similar strog association between multiple parities and development of cesarean sections have been reported in the study of Faiz ${ }^{(1)}$, Halimi ${ }^{(16)}$ and Shaikh ${ }^{(17) .}$

Ante-partum hemorrhage was expressed by 11 out of $20(55 \%)$ of placenta previa group in the current study. It was of the profuse form in 2 out of these 11 patients. Ante partum bleeding was considered a statistically significant association with Placenta previa . this agrees with the reports of Burd (2012) ${ }^{18}$ and Fan Dazhi (2017) ${ }^{19}$, who reported incidences of $51.6 \%$ to $93 \%$ of antepartum bleeding in cases of PP .

US has been reported to be valuable technique in diagnosis and assessment of placenta previa using trans abdominal and trans-vaginal approaches ${ }^{(2-7}$ \& 20). Although MRI has been found to be superior to US especially in assessment of PP associated with complications like placenta accrete, et its high cost and limited availability make MRI not $\operatorname{popular}^{(15)}$

TVUS was found to be tolerated by all pregnant women in current study with no associated complications during examination comprising vaginal bleeding, abortions nor iatrogenic rupture of membranes. Farine 1988 reported similar findings regarding the occurrence of fetal or maternal complications with the use of TVUS during pregnancy ${ }^{(5)}$.

In the present study, there were no false positive results by transvaginal ultrasound compared to (2/40) 5\% false positive results of trans-abdominal ultrasound. TAUS overestimated the diagnosis of placenta previa in two pregnant women. One of them was reported as PP marginalis and the other was low lying placenta. Both TVUS and operative room results, disapproved these findings and reported both cases as having normal relation between the lower edge of the placenta and the internal os. No false negative diagnosis was reported by both TAUS and TVUS in the current study. Both techniques showed identical sensitivities of $100 \%$. The specificity was $90 \%$ \&
$100 \%$ for the TAUS and TVUS respectively. The positive predictive value was $90 \%$ \& $100 \%$ for TAUS and TVUS respectively. The negative predictive value was $0 \%$ for both techniques. Subsequently TVUS proved to be more accurate in detection of relation of the lower edge of placenta to the internal os. In 1988, Farine ${ }^{(5)}$ studied the role of vaginal ultrasound for diagnosis of placenta previa. The exact location of the internal os was defined in all women by trans-vaginal root compared to only 36? 55 women by the trans abdominal root. They reported that TVUS correctly diagnosed 39/45 PP cases compared to the TAUS which only did so in 20/45 women having placenta previa. TVUS was reported to have $100 \%$ sensitivity and $88 \%$ specificity with negative predictive value of $100 \%$.

Sherman (1992) compared the diagnostic yields between TAUS and TVUS in assessment of $\mathrm{PP}^{(21)}$. Similar to the current study findings, they reported up to $6 \%$ false positive results of TAUS and false negative results of $2 \%$. The false positive and negative results were absent in their cases studied with TVUS as it raised the clarity of demonstrating the lower edge of the placenta in relation to the internal os especially in those who have posterior placenta previa.

Also, Oppenheimer (2007) $)^{(20)}$ reported near similar results to ours, including $7 \%$ false positive and $0 \%$ false negative results of TAUS in diagnosis of placenta previa. Their findings by TVUS were quite identical to those of operating room.

Despite these promising results, TVUS is not preferred by many Egyptian patients especially those with vaginal bleeding. Some obstetricians have also a concept to contraindicate TVUS for those women who have vaginal bleeding, This was the major obstacle in collecting data in the current study

\section{Conclusion}

Final diagnosis of placenta previa should be established on the $3^{\text {rd }}$ trimester. 
Transabdominal ultrasound has an inherent advantage of concurrent assessment of gestational age, fetal well-being, the exclusion of congenital anomalies and placental localization. However, it is associated with higher false positive results for diagnosis of placenta previa in comparison to transvaginal ultrasound.

Transvaginal ultrasound is a simple \& safe method, despite causing little discomfort to the patients. It is a valuable procedure to compliment transabdominal ultrasound in the evaluation of patients with suspected placenta previa and also helps to exclude false positives and to determine the mode of delivery, although it cannot replace transabdominal so should be incorporated routinely when transabdominal visualization of the placenta is not conclusive.

\section{References}

1. Faiz AS, Ananth CV. Etiology and risk factors for placenta previa: an overview and meta-analysis of observational studies. J Matern Fetal Neonatal Med 2003; 13: 175-90.

2. Wexler P, Nerons KR. Early diagnosis of placenta previa. Obstet Gynecol. 2007; 54(2):231-4.

3. Weerasekera DS. Placenta praevia and scarred uterus - an obstetrician's dilemma. J Obstet Gynaecol 2000; 5: 484-5.

4. Lahoria K, Malhotra S, Bagga R. Transabdominal and transvaginal ultrasonography of placenta previa. Int $\mathbf{J}$ Gynaecol Obstet 2007;98(3):258-259 .

5. Farine D, Ritchie $\mathrm{J}$ K. What is a low lying placenta? Am J Obstet Gynecol 1988;165(4):1036-1038.

6. Howerd T H, Catherine MM, Robert GW, Rosemary GR, Carol BB. Outcomes of pregnancies with low lying placenta diagnosed in the second trimester sonography. J Ultrasound Med 2014;33: 691-696

7. Blouin D,Rioux C Routine third trimester control ultrasound examination for low lying or marginal placentas diagnosed at mid pregnancy: is this indicated?. J Obstet Gynaecol Can 2012;34:425-428

8. Baughman WC, Corteville J E, Shah RR. Placenta accreta: spectrum of US and MR imaging findings: Radiographics. 2012; 1905: 1960-16.

9. Rose AA, Gopalan $U$ correlation of maternal age with placenta previa. Int $\mathrm{J}$ Med Res Rev 2015;3(9):914-918

10. Tuzović L, Djelmis J, Ilijić M. Obstetric risk factors associated with placenta previa development: case-control study. Croat Med J. 2003 Dec;44(6):728-33.

11. Uyanikoglu H, Karaham MA, Trup AB, Agar M, Tasduzen ME Are multiple repeated cesarean sections really as safe? Turkish J Matern Fetal Neonatal Med 2017; 30(4):482-485

12. Ananth CV, Wilcox AJ, Savitz DA, Bowes WA Jr, Luther ER,. Effect of maternal age and parity on the risk of utero-placental bleeding disorders in pregnancy. Obstet Gyneacol 1996;88(4):512-516

13. Milosevic J, Lilic V,Tasic M, RadovicJanosevic D, Stefanovic M, Antic V Pacental complications after a previous cesarean section. Med Pregl 2009;62(56):212-216

14. Itedal A, Qurashi M, Moawia A, Sayed M Association of cesarean section with placenta previa IOSR-JDMS 2015;29:3234

15. David AC, Edozein LC, Smith GC, Mahmood AT, Allan Templeton A. Risk of placenta previa in 2nd birth after fist birth cesarean section: A population based study and meta analysis Utrasound Obstet Gynaecol 2011;11:95

16. Halimi S. Association of placenta previa with multiple parities JPMI 2011;139 (2): 142

17. Shaikh S. Frequency of placenta previa in multigravida at tertiary care hospital. Int $\mathbf{J}$ Cur Res Rev 2014;39:42-43 
18. Burd I. The incidence of vaginal bleeding in cases of placenta previa. Am J Obstet Gynaecol 2012;18:23

19. Dazhi Fan LU, Song WU, Qing XA, Wang W, Xiaoling GU Prevalence of antepartum hemorrhage in women with placenta previa: A systemic review and meta analysis. Sci Rep 2017;40:48-47

20. Oppenheimer L. Diagnosis and management of placenta previa. J Obstet Gynaecol Can. 2007; 29:261-73.

21. Sherman SJ, Carlson DE, Platt LD, Medearis AL. Transvaginal ultrasound : Does it help in diagnosis of placenta previa? Ultrasound Obstet Gynaecol 1992;2(4):256-260. 